\title{
Axisymmetric Alfvén resonances in a multi-component plasma at finite ion gyrofrequency
}

\author{
D. Yu. Klimushkin ${ }^{1}$, P. N. Mager ${ }^{1}$, and K.-H. Glassmeier ${ }^{2}$ \\ ${ }^{1}$ Institute of Solar-Terrestrial Physics (ISTP), Russian Academy of Science, Siberian Branch, Irkutsk, P.O.Box 4026, 664033, \\ Russia \\ 2 Institut für Geophysik und Extraterrestrische Physik, Mendelssohnstr. 3, D-38106 Braunschweig, Germany
}

Received: 8 August 2005 - Revised: 15 February 2006 - Accepted: 16 February 2006 - Published: 19 May 2006

\begin{abstract}
This paper deals with the spatial structure of zero azimuthal wave number ULF oscillations in a 1-D inhomogeneous multi-component plasma when a finite ion gyrofrequency is taken into account. Such oscillations may occur in the terrestrial magnetosphere as Pc1-3 waves or in the magnetosphere of the planet Mercury. The wave field was found to have a sharp peak on some magnetic surfaces, an analogy of the Alfvén (field line) resonance in one-fluid MHD theory. The resonance can only take place for waves with frequencies in the intervals $\omega<\omega_{c h}$ or $\Omega_{0}<\omega<\omega_{c p}$, where $\omega_{c h}$ and $\omega_{c p}$ are heavy and light ions gyrofrequencies, and $\Omega_{0}$ is a kind of hybrid frequency. Contrary to ordinary Alfvén resonance, the wave resonance under consideration takes place even at the zero azimuthal wave number. The radial component of the wave electric field has a pole-type singularity, while the azimuthal component is finite but has a branching point singularity on the resonance surface. The later singularity can disappear at some frequencies. In the region adjacent to the resonant surface the mode is standing across the magnetic shells.
\end{abstract}

Keywords. Magnetospheric physics (MHD waves and instabilities; Planetary magnetospheres) - Space plasma physics (kinetic and MHD theory)

\section{Introduction}

This paper is devoted to the study of resonant excitation of ultra low frequency (ULF) waves in an inhomogeneous multicomponent plasma. Alfvén resonances, also called field line resonances, are one of the key physical processes in the magnetosphere of the Earth (Tamao, 1965; Hasegawa and Chen, 1974; Southwood, 1974) and other planets (Khurana, 1993;

Correspondence to: D. Klimushkin

(klimush@iszf.irk.ru)
Glassmeier et al., 1999, 2004; Othmer et al., 1999). Treatment of this phenomenon in a one-dimensional inhomogeneous model of the magnetosphere, using a one-fluid MHD plasma model, is as follows. A fast magnetosonic (FMS) wave propagates into the inhomogeneous magnetosphere and reflects from a particular magnetic surface. The superposition of ingoing and outgoing waves forms a standing wave pattern in the FMS transparent region. However, part of the FMS energy penetrates through this boundary (resembling the tunneling effect in quantum mechanics) and excites the Alfvén wave close to a surface, where the wave frequency equals the local Alfvén frequency $\Omega=v_{A} k_{\|}$, where $v_{A}=B / \sqrt{4 \pi \rho}$ is the Alfvén velocity, $B$ the equilibrium magnetic field, and $\rho$ the background plasma density. The azimuthal and radial components of the wave electric field exhibits a logarithmic or pole-type singularity at the resonance surface, respectively. All of the FMS wave energy is absorbed near this Alfvén resonance surface. A more detailed theoretical analysis indicates that axisymmetric oscillations with azimuthal wave numbers $m=0$ cannot experience this Alfvén resonance.

However, this standard theoretical treatment of the Alfvén resonance, based on a one-fluid MHD plasma model, assumes the wave frequency $\omega$ to be infinitely small compared to the thermal ion gyrofrequency $\omega_{c i}$. If the ratio $\omega / \omega_{c i}$ is finite, the resonant excitation of Alfvén waves via coupling to FMS waves is possible even for the special case $m=0$ (e.g. Leonovich et al., 1983; Timofeev, 1992; Glassmeier et al., 2003). This is due to the fact that at finite gyrofrequencies and assuming a vanishing electric field along the background magnetic field, the MHD wave dispersion relation becomes (e.g. Swanson, 1989, Eq. 2.16):

$$
\left(\frac{\omega^{2}}{c^{2}} \varepsilon_{\perp}-k_{\|}^{2}\right)\left(\frac{\omega^{2}}{c^{2}} \varepsilon_{\perp}-k_{\|}^{2}-k_{\perp}^{2}\right)=\frac{\omega^{4}}{c^{4}} \eta^{2},
$$

Published by Copernicus GmbH on behalf of the European Geosciences Union. 
where $k_{\|}$, and $k_{\perp}$ are the wave vector components parallel and transverse to the ambient magnetic field, $\varepsilon_{\perp}$ is the diagonal element of the dielectric tensor, and $\eta$ its off-diagonal element proportional to the ratio $\omega / \omega_{c i}$. The effect of heavy ions on ULF waves has been recently studied by Kim and Lee (2004).

In the terrestrial magnetosphere, finite $\omega / \omega_{c i}$ effects can be important for Pc1-2 or Pi1 pulsations, especially if the magnetosphere is enriched with heavy (oxygen) ions, as may occur during substorm activity. These effects can also play an essential role in ULF wave phenomena in other magnetospheres. Indeed, in Mariner 10 data, Russell (1989) found magnetic pulsations in the magnetosphere of the planet Mercury with a period of about $2 \mathrm{~s}$. This period is only a bit larger than the cyclotron period of hydrogen ions and less than the cyclotron period of sodium ions, which are abundant in this magnetosphere (Ip, 1986; Cheng et al., 1987; Killen and Ip, 1999). Othmer et al. (1999) interpreted the micropulsation observed by Russell (1989) as the analog of a field line resonance in the multi-component plasma of the Hermean magnetosphere.

The aim of this paper is to study in detail the spatial structure of ULF waves with a zero azimuthal wave number in a one-dimensional inhomogeneous, multi-component space plasma with a finite ratio $\omega / \omega_{c i}$. In Sect. 2 we derive the governing wave equations while in Sect. 3 we suggest a possible solution in the WKB-approximation. Section 4 is devoted to a more detailed study of resonance conditions in multi-component plasmas. The main results of our study are presented in Sect. 5.

\section{The wave equation}

We investigate a box model of the magnetosphere where field lines are straight and parallel. The model is one-dimensional and inhomogeneous, all equilibrium parameters depend only on one coordinate, $x$, imitating the radial coordinate in the magnetosphere. The coordinate $y$ is directed in the azimuthal direction, while the $z$ coordinate is directed along the ambient magnetic field lines. A cold plasma is assumed, that is the plasma pressure is zero. The plasma is composed of electrons, light ions (protons), and heavy ions.

From Maxwell equations we obtain the equation for the electric field $\boldsymbol{E}$ of the wave with frequency $\omega$ :

$\frac{\omega^{2}}{c^{2}} \hat{\varepsilon} \boldsymbol{E}=\nabla \times \nabla \times \boldsymbol{E}$,

where the dielectric tensor elements

$\hat{\varepsilon}=\left(\begin{array}{ccc}\varepsilon_{\perp} & -i \eta & 0 \\ i \eta & \varepsilon_{\perp} & 0 \\ 0 & 0 & \varepsilon_{\|}\end{array}\right)$ in the ULF range $\left(\omega \ll \omega_{c e}\right)$ become (e.g. Glassmeier et al., 2003):

$$
\begin{aligned}
& \varepsilon_{\|}=-\infty, \\
& \varepsilon_{\perp}=\sum_{i} \frac{\omega_{p i}^{2}}{\omega_{c i}^{2}-\omega^{2}}, \\
& \eta=\frac{\omega_{p e}^{2}}{\omega \omega_{c e}}-\sum_{i} \frac{\omega_{c i}}{\omega} \frac{\omega_{p i}^{2}}{\omega_{c i}^{2}-\omega^{2}} .
\end{aligned}
$$

Here $\omega_{p}$ and $\omega_{c}$ are the plasma and cyclotron frequencies of electrons (index " $e$ ") and ions (index " $i$ "), respectively. The infinite value of the dielectric tensor longitudinal element implies a vanishingly small value for the longitudinal electric field component of the wave. Note that in cases where the wave frequency is much lower than the gyrofrequency of any ion fraction the dielectric tensor transverse elements take the form

$\varepsilon_{\perp}=\frac{c^{2}}{v_{A}^{2}}, \quad \eta=\sum_{i} \frac{\omega}{\omega_{c i}} \frac{c^{2}}{v_{i A}^{2}}$,

where $v_{i A}=B / \sqrt{4 \pi \rho_{i}}$ is the Alfvén velocity determined by the $i^{t h}$ ion component.

We assume that the tensor elements $\varepsilon_{\perp}$ and $\eta$ depend only on the radial coordinate $x$. The wave field is assumed to vary in the $y$ and $z$ directions as $e^{i k_{y} y+i k_{z} z}$, where $k_{y}$ is the analog of the azimuthal wave number $m$ for the box model.

With these definitions we obtain from Eq. (2):

$$
\begin{aligned}
& \frac{c^{2}}{\omega^{2}}\left(\varepsilon_{\perp} E_{x}-i \eta E_{y}\right)=i k_{y}\left(\frac{d E_{y}}{d x}-i k_{y} E_{x}\right)+k_{z}^{2} E_{x}, \\
& \frac{c^{2}}{\omega^{2}}\left(i \eta E_{x}+\varepsilon_{\perp} E_{y}\right)=\frac{d}{d x}\left(i k_{y} E_{x}-\frac{d E_{y}}{d x}\right)+k_{z}^{2} E_{y} .
\end{aligned}
$$

\section{Let us designate}

$K=\frac{\omega^{2}}{c^{2}} \varepsilon_{\perp}-k_{z}^{2}$.

In the $\omega \ll \omega_{c i}$ range, this value takes the form $K=\left(\omega / v_{A}\right)^{2}-k_{z}^{2}$. Outside the Earth's plasmapause, $K$ is increasing with distance from the Earth because the Alfvén velocity is a monotonically decreasing function of $x$. We will suppose the same behavior of $K$ in our multi-component model, that is $K^{\prime}>0$, where the prime denotes differentiation with respect to $x$. Moreover, we will assume that it becomes constant for $x \rightarrow \pm \infty$.

Expressing $E_{x}$ from Eq. (3) and substituting it into Eq. (4), we yield the equation for the azimuthal component of the electric field:

$$
\begin{array}{r}
E_{y}^{\prime \prime}-k_{y}^{2} \frac{\omega^{2}}{c^{2}} \frac{\varepsilon_{\perp}^{\prime} E_{y}^{\prime}}{K\left(K-k_{y}^{2}\right)}+\left[K-k_{y}^{2}-\frac{\omega^{4}}{c^{4}} \frac{\eta^{2}}{K}-\right. \\
\left.k_{y} \frac{\omega^{4}}{c^{4}} \frac{\eta \varepsilon_{\perp}^{\prime}}{K\left(K-k_{y}^{2}\right)}+k_{y} \frac{\omega^{2}}{c^{2}} \frac{\eta^{\prime}}{K}\right] E_{y}=0 .
\end{array}
$$




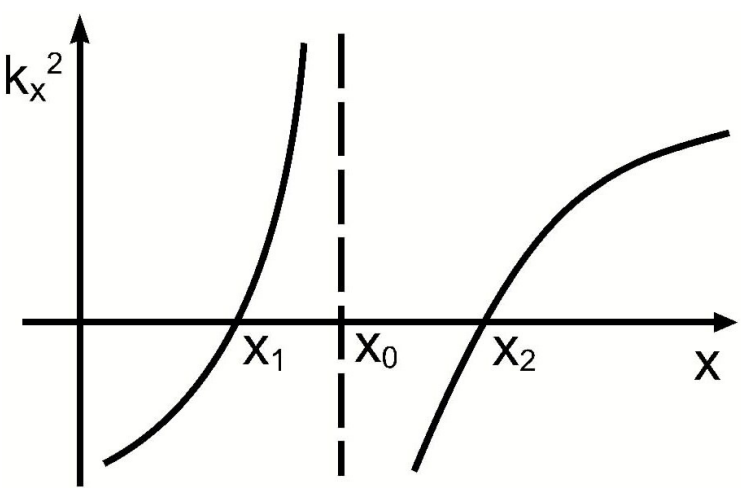

Fig. 1. The wave vector radial component squared, $k_{x}^{2}$.

In a homogeneous plasma this equation reduces to the dispersion relation (1). For $\omega \ll \omega_{c i}$, Eq. (5) reduces to Eq. (2) from Southwood (1974).

For axisymmetric oscillations with $k_{y}=0$ Eq. (5) reduces to

$E_{y}^{\prime \prime}+\left[K-\frac{\omega^{4}}{c^{4}} \frac{\eta^{2}}{K}\right] E_{y}=0$.

It is this equation that we would like to discuss here. It was already discussed in a more general way by Glassmeier et al. (2003). Note that for the special case $\eta=0$ it reduces to the box model analog of Dungey's (1954) decoupled toroidal electric field equation.

The electric field radial component is expressed through the azimuthal component as

$E_{x}=\frac{\omega^{2}}{c^{2}} \frac{i \eta E_{y}}{K}$.

As a boundary condition we use the boundedness of the function $E_{y}$ as $x \rightarrow \pm \infty$.

\section{The structure of the wave field}

Equation (6) can be solved within the WKB approximation. Let us designate

$U=K(x)-\frac{\omega^{4}}{c^{4}} \frac{\eta^{2}(x)}{K(x)}$.

Then the radial component of the wave vector is determined by the equality

$k_{x}=\sqrt{U}$

The variation of $k_{x}^{2}$ with $x$ is schematically displayed in Fig. 1.

Near the points $x_{0}, x_{1}$, and $x_{2}$ the WKB approximation cannot be used as $k_{x}$ approaches infinity at the singular turning point $x_{0}$ and becomes zero at the regular turning points $x_{1,2}$. Transparent regions are determined by the condition

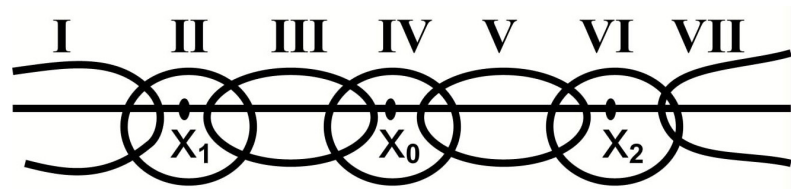

Fig. 2. The regions where the solutions of the wave equation have been determined.

$k_{x}^{2}>0$. They are located between the points $x_{1}$ and $x_{0}$ as well as at $x>x_{2}$.

We now introduce intersecting regions I-VII with respect to the radial coordinate (Fig. 2). Regions I, III, V, VII are located far enough away from the turning points where the WKB ansatz is applicable. In regions II, IV, VI it is possible to use the linear expansion of the function $K(x)$. With this assumption Eq. (6) reduces to an Airy differential equation in regions II and VI. These regions intersect with regions I, III and V, VII, respectively. In region IV, left (right) from the point $x_{0}$, Eq. (6) is reduced to a Bessel differential equation (modified Bessel equation) of the 1st order. This region intersects with regions III and V.

Matching solutions in the intersecting regions, we find the full solution of the original differential equation. The only undetermined parameter is the constant $a$, representing the wave amplitude of the $E_{y}$ component. We shall present solutions only in regions II-VI, omitting intermediate mathematical details in the Appendix. For region II we find:

$E_{y}=a \operatorname{Ai}\left(\frac{x_{1}-x}{\lambda_{1}}\right)$.

In region III we have:

$E_{y}=\frac{a}{\sqrt{k_{x}}} \frac{\left(U_{1}^{\prime}\right)^{1 / 6}}{\sqrt{\pi}} \sin \left[\int_{x_{1}}^{x} k_{x}\left(x^{\prime}\right) d x^{\prime}+\frac{i \pi}{4}\right]$.

The solution in region IV for $x<x_{0}$ yields

$$
\begin{aligned}
E_{y} & =-a\left(U_{1}^{\prime}\right)^{1 / 6} \sqrt{x_{0}-x}\left[\cos \varphi \cdot \mathrm{J}_{1}\left(2 \sqrt{\frac{x_{0}-x}{\lambda_{0}}}\right)\right. \\
& \left.+\sin \varphi \cdot \mathrm{Y}_{1}\left(2 \sqrt{\frac{x_{0}-x}{\lambda_{0}}}\right)\right]
\end{aligned}
$$

while in the same region, but for $x>x_{0}$ we have:

$$
\begin{aligned}
E_{y} & =a\left(U_{1}^{\prime}\right)^{1 / 6} \sqrt{x-x_{0}}\left[e^{-i \varphi} \cdot \mathrm{I}_{1}\left(2 \sqrt{\frac{x_{0}-x}{\lambda_{0}}}\right)\right. \\
& \left.+\frac{2}{\pi} \sin \varphi \cdot \mathrm{K}_{1}\left(2 \sqrt{\frac{x_{0}-x}{\lambda_{0}}}\right)\right] .
\end{aligned}
$$

The region $\mathrm{V}$ solution reads:

$$
\begin{aligned}
E_{y} & =\frac{1}{\sqrt{\left|k_{x}\right|}} \frac{a}{\sqrt{\pi}}\left(U_{1}^{\prime}\right)^{1 / 6}\left[\frac{1}{2} \exp \left(-i \varphi+\int_{x_{0}}^{x}\left|k_{x}\right| d x^{\prime}\right)\right. \\
& \left.+\sin \varphi \exp \left(-\int_{x_{0}}^{x}\left|k_{x}\right| d x^{\prime}\right)\right] .
\end{aligned}
$$


And finally for region VI we obtain:

$$
\begin{aligned}
E_{y} & =a\left(\frac{U_{1}^{\prime}}{U_{2}^{\prime}}\right)^{1 / 6}\left[2 \sin \varphi e^{-\psi} \mathrm{Ai}\left(\frac{x_{2}-x}{\lambda_{2}}\right)\right. \\
& \left.+\frac{1}{2} e^{\psi-i \varphi} \operatorname{Bi}\left(\frac{x_{2}-x}{\lambda_{2}}\right)\right] .
\end{aligned}
$$

The following definitions and abbreviations have been used in the above expressions: $\mathrm{Ai}$ and $\mathrm{Bi}$ are Airy functions, $\mathrm{J}_{1}$ and $\mathrm{Y}_{1}$ denote Bessel functions, while $\mathrm{I}_{1}$ and $\mathrm{K}_{1}$ are modified Bessel functions, $U_{1,2}^{\prime}$ are the derivatives $d U / d x$ taken at the points $x_{1}, x_{2}$ (both are positive), $K_{0}^{\prime} \equiv d U\left(x_{0}\right) / d x, \lambda_{1,2}=\left(U_{1,2}^{\prime}\right)^{-1 / 3}, \lambda_{0}=c^{4} K_{0}^{\prime} / \omega^{4} \eta^{2}$, $\varphi=\int_{x_{1}}^{x_{0}} k_{x} d x$, and $\psi=\int_{x_{0}}^{x_{2}}\left|k_{x}\right| d x$.

Let us discuss the principal conclusions following from this solution. First of all, is there an analogy to the Alfvén resonance of MHD theory in this solution, that is, does the solution contain a singularity of the wave field? To answer this question, let us write the asymptotic of the expression (11) near the point $x_{0}$ (in the region $x<x_{0}$ ), where the function $K(\omega, x)$ goes to zero:

$$
\begin{aligned}
E_{y} & \simeq a \sqrt{\frac{\lambda_{0}}{\pi}}\left(U_{1}^{\prime}\right)^{1 / 6}\left[\operatorname { s i n } \varphi \cdot \left(1+2 \frac{x_{0}-x}{\lambda_{0}}\right.\right. \\
& \left.\left.\times \ln \sqrt{\frac{x_{0}-x}{\lambda_{0}}}\right)+O\left(\frac{x_{0}-x}{\lambda_{0}}\right)\right] .
\end{aligned}
$$

We see that the wave amplitude is finite near this point, but has a branching-point singularity. This behavior is strikingly different from the corresponding behavior for the ordinary Alfvén resonance, where the $E_{y}$ component is infinite, exhibiting the logarithmic singularity on the resonance surface. However, as is seen from Eq. (7), the radial component of the electric field has a pole-type singularity

$E_{x} \propto\left(x_{0}-x\right)^{-1}$

because the denominator $K$ can be expressed as $K \simeq K_{0}^{\prime}\left(x-x_{0}\right)$. This resembles the ordinary Alfvén resonance case.

But there are further differences. Note the factor $\sin \varphi$ in Eq. (15). It means that under the condition

$$
\int_{x_{1}}^{x_{0}} k_{x} d x=\pi n
$$

where $n$ is integer, this branching singularity disappears. Because all the values $x_{1}, x_{0}, k_{x}$ depend on $\omega$, Eq. (17) represents a kind of a quantization condition for the frequency $\omega$. If this condition is satisfied, the leading term in expression (15) becomes $x_{0}-x$; this terms cancels out in Eq. (7), and the pole-type singularity (16) disappears, too.

Yet another interesting feature can be found when we consider the time-averaged Poynting flux (e.g. Landau and Lifshitz, 1960)

$$
\langle\boldsymbol{S}\rangle=\frac{c}{8 \pi} \operatorname{Re}\left[\boldsymbol{E} \times \boldsymbol{b}^{*}\right]
$$

through the resonance surface (here $\boldsymbol{b}$ is magnetic field of the oscillation, asterisk means the complex conjugate). As is well known in the MHD case, the vector $\langle\boldsymbol{S}\rangle$ is directed toward the resonant surface at $x>x_{0}$, and it is zero at $x<x_{0}$, that is energy flows toward the resonance from the side of the FMS transparent region and is completely absorbed at this surface. In our case, taking the $x$-component of Eq. (18), expressing the magnetic field from the equation $(i \omega / c) \boldsymbol{b}=\boldsymbol{\nabla} \times \boldsymbol{E}$, and expressing the electric field near the resonance from Eqs. $(11,12)$, we find that

$\left\langle S_{x}\right\rangle=0$

at $x<x_{0}$, and

$\left\langle S_{x}\right\rangle=-\frac{c^{2} a^{2}}{8 \pi^{2} \omega}\left(U_{1}^{\prime}\right)^{1 / 3} \sin ^{2} \varphi$

at $x>x_{0}$. It resembles the situation in the MHD case, with an important difference: when $\sin \varphi=0$, that is condition (17) is satisfied, the Poynting flux through $x_{0}$ is zero, that is absorption of energy is absent. Of course, it is not unexpected, because there is no resonance in this case, as we already know.

Then, it follows from Eq. (10) that the wave is standing across magnetic shells in the transparent region $x_{1}<x<x_{0}$. This is a rather unexpected behavior because the transparent region is bounded with regular and singular turning points. It is important to note that this standing wave pattern appears at any wave frequency, and thus is it not associated with any quantization condition of the frequency $\omega$ or any other value. In the $\omega / \omega_{c i}=0$ case an oscillatory structure arises when the field line curvature (Leonovich and Mazur, 1993; Klimushkin, 1998) or the magnetic field shear (Klimushkin and Mager, 2004) are taken into account, but in these cases the wave is travelling rather than standing across magnetic shells.

As can be seen from Eq. (14), the asymptotic behavior of the $E_{y}$ component in the region $\left(x-x_{2}\right) / \lambda_{2} \gg 1$, that is in the transparent region $x>x_{2}$ immediately adjacent to the point $x_{2}$, is described by the expression

$$
\begin{aligned}
E_{y} & \simeq \frac{a}{\sqrt{\pi}}\left(\frac{U_{1}^{\prime}}{U_{2}^{\prime}}\right)^{1 / 6}\left(\frac{\lambda_{2}}{x-x_{2}}\right)^{1 / 4} \\
& \times\left[2 e^{-\psi} \sin \varphi \sin \left(\int_{x_{2}}^{x} k_{x} d x^{\prime}+\frac{\pi}{4}\right)\right. \\
& \left.+\frac{1}{2} e^{\psi-i \varphi} \cos \left(\int_{x_{2}}^{x} k_{x} d x^{\prime}+\frac{\pi}{4}\right)\right] .
\end{aligned}
$$

This describes the superposition of two standing waves with very different ( $e^{-2 \psi}$ times) amplitudes. It also can be represented as a superposition of a wave traveling towards the point $x_{2}$ and a wave reflected there. The amplitude of the outgoing wave is a bit smaller than that of the ingoing wave, due to absorption of part of the wave energy at the resonance point. This is not an unusual behavior, and it also characterizes the wave field structure in a one-fluid MHD approximation. There is an essential difference, however: when 
$\sin \varphi=0$, that is, at the very condition (17) leading to the disappearance of the singularities (15) and (16), the first (small amplitude) standing wave disappears, which means that ingoing and outgoing waves have the same amplitudes. In this case, no absorption of energy into the resonance occurs.

From an observational point of view it is interesting to know the change in the polarization of the wave field as a function of position. For the special case $k_{y}=0$ one can easily derive the following expression for the electric field polarization from Eq. (7):

$$
\frac{E_{x}}{E_{y}}=\frac{\omega^{2}}{c^{2}} \frac{i \eta}{K} .
$$

A nonzero and finite value of this ratio implies an elliptic polarization, while the cases $E_{x} / E_{y}=0$ and $E_{x} / E_{y} \rightarrow \infty$ describe linear polarization. Thus, at the resonance surface $x=x_{0}$ the wave exhibits a linear polarization, because at this position $K=0$. The sign of the polarization changes across this surface, which corresponds to a change in the phase of the wave by $180^{\circ}$. Again, the same behavior is a property of the MHD Alfvén resonance (Hasegawa and Chen, 1974; Southwood, 1974). But there are some important differences. In the present case the change in the sign of the ratio $E_{x} / E_{y}$ is not connected with the change in the sign of the derivative $d E_{y} / d x$, as in $\omega / \omega_{c i}=0$ case. Another difference is the dependence of $E_{x} / E_{y}$ on the $k_{y}$ value in the MHD case.

\section{Resonance frequencies in a multi-component plasma}

Othmer et al. (1999) have discussed in detail resonance problems in a multi-component plasma. They proposed that resonant mode coupling should preferentially occur at the crossover frequency

$\Omega_{C R}^{2}=\left(1+\frac{n_{h}}{n_{p}}\right)^{-1} \cdot\left(\omega_{c h}^{2}+\frac{n_{h}}{n_{p}} \omega_{c p}^{2}\right)$,

determined by the condition

$\eta\left(\omega=\Omega_{C R}\right)=0$.

Here $n$ denotes the number density and indices $p$ and $h$ stand for light ions (protons) and heavy ions.

For this special case the dielectric tensor is a purely diagonal tensor much as in the MHD case. Thus, at the cross-over frequency $\Omega_{C R}$ there exists a strictly guided mode similar to the MHD Alfvén mode. Coupling into this mode should be very effective, as the energy coupled into this mode is not re-radiated away from the resonant magnetic shell due to offangle energy transport.

Here, we can also discuss such resonant mode coupling effects using the theoretical framework outlined above. Let us consider the resonance condition

$K(\omega, x) \equiv \frac{\omega^{2}}{c^{2}} \varepsilon_{\perp}(\omega, x)-k_{z}^{2}=0$.
And let $\Omega$ be a solution of this equation. The position of the resonance surface is determined as a solution of the equation $\omega=\Omega(x)$ with respect to the radial coordinate. The wave vector can be decomposed into a transverse and longitudinal component, $k_{\perp}$ and $k_{\|}$, respectively with $k^{2}=k_{\perp}^{2}+k_{\|}^{2}$ (here $k_{\perp}^{2} \equiv k_{x}^{2}+k_{y}^{2}, k_{\|} \equiv k_{z}$ ). In addition to condition (23), we require the longitudinal propagation condition $k_{\|}^{2} \geq 0$, as for $k_{\|}^{2}<0$ the wave structure along the ambient magnetic field would be evanescent. Note that, although near the resonance point $k_{\perp} \gg k_{\|}$, the value of the wave vector's transverse component $k_{\perp}$ does not enter into the resonance condition $K=0$. Furthermore, the value $\eta$, the off-diagonal components of the dielectric tensor, does not enter into the condition (23).

Defining a longitudinal refraction index $N_{\|}^{2}=k_{\|}^{2} c^{2} / \omega^{2}$ we can study the dependence of this refraction index on the wave frequency. For a plasma with two ion fractions, protons (in$\operatorname{dex} p$ ) and heavy ions (index $h$ ), the expression for the longitudinal refraction index becomes

$N_{\|}^{2}=\frac{\omega_{p p}^{2}}{\omega_{c p}^{2}-\Omega^{2}}+\frac{\omega_{p h}^{2}}{\omega_{c h}^{2}-\Omega^{2}}$.

The dependence $N_{\|}^{2}(\Omega)$ is shown in Fig. 3 and indicates that $N_{\|}^{2} \rightarrow-\infty$, if $\Omega \rightarrow \omega_{c h}$, and $N_{\|}^{2} \rightarrow+\infty$, if $\Omega \rightarrow \omega_{c p}$. In the frequency interval between the light and heavy ion gyrofrequencies there is a frequency $\Omega_{0}$, which corresponds to the value $N_{\|}^{2}=0$. It equals

$\Omega_{0}^{2}=\left(1+\frac{n_{h}}{n_{p}} \frac{m_{p}}{m_{h}}\right)^{-1} \cdot\left(\omega_{c h}^{2}+\frac{n_{h}}{n_{p}} \frac{m_{p}}{m_{h}} \omega_{c p}^{2}\right)$,

where $n_{h, p}$ are concentrations of heavy and light ions, and $m_{h, p}$ are their masses. As seen from Fig. 3, $N_{\|}^{2}>0$ only if $\Omega<\omega_{c h}$ or $\Omega_{0}<\Omega<\omega_{c p}$. Thus, resonance only occurs for waves with frequencies lying in these two allowed intervals. Waves in the first interval are usual ion-cyclotron waves, which become Alfvén waves in the limit $\omega / \omega_{c h} \rightarrow 0$. As for the waves in the second range, they do not have an analogy in an electron-proton plasma. The smaller the fraction is of heavy ions $n_{h} / n_{p}$, the closer the parallel cutoff frequency $\Omega_{0}$ to the heavy ion gyrofrequency, and the narrower the forbidden range $\omega_{c h}<\Omega<\Omega_{0}$. In the limit $n_{h} / n_{p} \rightarrow 0$, both allowed intervals are merged.

For the Hermean magnetosphere, according to estimates by Ip (1986) and Cheng et al. (1987), the sodium ions are abundant with $0.1<n_{N a} / n_{p}<1$ and $m_{N a} / m_{H}=23$; hence, the first term in this expression is only slightly different from unity. Note that the frequency of the pulsation registered by Mariner 10 in the Hermean magnetosphere (Russell, 1989) falls into the second range. As Othmer et al. (1999) argued that the cross-over frequency is a preferred frequency for resonant mode coupling, we need to compare both, the crossover frequency $\Omega_{C R}$ and the parallel cutoff frequency $\Omega_{0}$. For Hermean conditions $0.1<n_{N a} / n_{p}<1$ and $m_{N a} / m_{H}=23$ the cross-over frequency is always larger than the parallel 


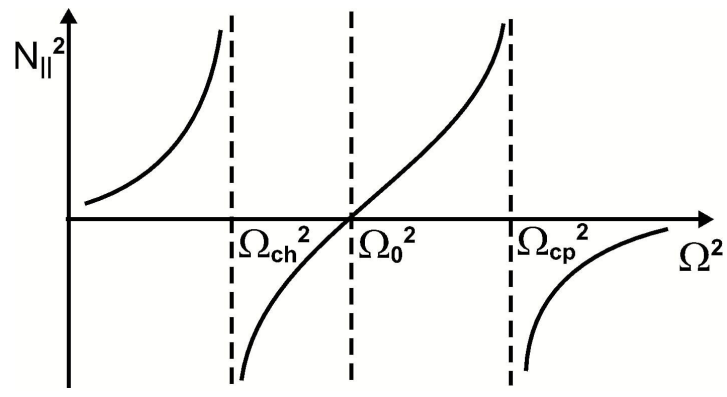

Fig. 3. The longitudinal refraction index as a function of wave frequency.

cutoff frequency. Thus, identification of $\Omega_{C R}$ as a preferred frequency for resonant mode coupling is not contradicted by our present results.

The terrestrial magnetosphere is enriched with oxygen ions during substorms. According to Wedeken et al. (1984), $n_{O} / n_{p} \sim 1 / 15$, which implies $\Omega_{0} \sim 0.1 \omega_{c p}$. Near the geomagnetic equator, the proton gyrofrequency can be estimated as $\omega_{c p} \sim 0.2 \mathrm{~s}^{-1}$, that is, some Pc3 pulsations (periods from 10 to $45 \mathrm{~s}$ ) fall into the second interval, $\Omega_{0}<\Omega<\omega_{c p}$, that is the range where one accounts for the finite gyrofrequency effects.

\section{Conclusions}

This work is concerned with the axisymmetric ULF oscillations in a one- dimensional, inhomogeneous, multicomponent plasma with a finite ratio of wave frequency and ion gyrofrequency. Such conditions will occur in the magnetosphere of Mercury, and in the terrestrial magnetosphere, if it is enriched with oxygen ions due to substorm activity. Even in the case of $k_{y}=0$, this finite ratio leads to a wave resonance that, to some extent, resembles the classical Alfvén resonance. The following picture emerges from our study: a wave propagating across magnetic field lines in the inhomogeneous plasma reflects at some boundary. The superposition of ingoing and outgoing waves forms a standing wave, but part of the energy percolates deeper into the magnetosphere and excites a mode sharply localized near the resonance surface at the special position $x_{0}$, determined by the relation $\left(\omega^{2} / c^{2}\right) \varepsilon_{\perp}\left(x_{0}\right)-k_{\|}^{2}=0$. As in the case of the classical Alfvén resonance, the radial component of the wave electric field exhibits a singularity $E_{x} \propto\left(x-x_{0}\right)^{-1}$. The resonantly generated wave acts back on the generating wave, resulting in a small difference between the ingoing and outgoing waves' amplitudes, that is part of the wave energy is absorbed in the resonance surface.

However, there are many differences between the resonance in the $k_{y} \neq 0, \omega / \omega_{c i}=0$ case, on the one hand, and the $k_{y}=0, \omega / \omega_{c i} \neq 0$ case, on the other hand. In the former case, that is in the ordinary or classical Alfvén resonance, the azimuthal component of the electric field has a logarithmic singularity and infinite, $E_{y} \propto \ln \left(x-x_{0}\right)$, while in the latter case the azimuthal component is finite on the resonance surface, but has a branching point singularity.

Moreover, for certain frequencies defined by Eq. (17) these singularities in $E_{y}$ and $E_{x}$ can disappear. Then, the resonance surface is immediately adjacent to the mode transparent region, where the wave vector radial component squared is positive. The wave is standing across magnetic shells in this region. There is no analogy of this transparent region in the model with straight parallel field lines in the case $\omega / \omega_{c i}=0$, where the very notion of the wave vector radial component is applicable only in the fast magnetosonic mode localization region.

Detailed consideration of the resonance condition shows that resonance can occur for only waves with frequencies in the ranges $\omega<\omega_{c h}$ or $\Omega_{0}<\omega<\omega_{c p}$, where $\omega_{c h}$ and $\omega_{c p}$ are gyrofrequencies of heavy and light ions, and $\Omega_{0}$ is a hybrid frequency. Waves in the first interval are an analogy of ioncyclotron waves which convert into Alfvén waves in the limit $\omega / \omega_{c h} \rightarrow 0$. Waves in the second interval do not have analogies in the electron-proton plasma. The Hermean pulsation event identified by Russell (1989) and some Pc3 pulsations in the terrestrial magnetosphere fall into this range.

The box-model considered in the present paper is certainly oversimplified, as planetary magnetospheres are essentially inhomogeneous along magnetic field lines. As for the ordinary field-line resonance, the influence of the parallel inhomogeneity was studied by, for example, Southwood and Kivelson (1986), Chen and Cowley (1989), Leonovich and Mazur (1989, 1993), and Fedorov et al. (1995). The general result is that the wave field global structure qualitatively is the same as in the 1-D inhomogeneous case, at least for small azimuthal wave numbers (for high $m$ numbers, see Leonovich and Mazur, 1993). As for multi-component plasmas, essential differences can be expected due to gyrofrequency changes along the field line, so moving along a field line we must intersect a point where $\omega=\omega_{c}$, at least for sodium ions. This can lead to changes in the parallel cutoff frequency $\Omega_{0}$ along the field line; in particular, points with $\Omega_{0}=0$ and $\Omega_{0} \rightarrow \infty$ can appear. A more detailed discussion of this effect is the subject of future work.

\section{Appendix A}

\section{Some details of the WKB solution}

Here we present some details of the solution of Eq. (6).

First, we consider the region II, where this equation takes the form

$E_{y}^{\prime \prime}+U_{1}^{\prime} \cdot\left(x-x_{1}\right) E_{y}=0$. 
Its general solution is

$$
E_{y}=a_{I I}^{1} \operatorname{Ai}(\xi)+a_{I I}^{2} \operatorname{Bi}(\xi),
$$

where $\xi=-\left(x-x_{1}\right) / \lambda_{1}$ and $a_{I I}^{1,2}$ are indefinite constants. The first term, Ai, is exponentially decaying to the left of the point $x_{1}$, while the second one grows. The region II gradually transforms into the region I without any reflecting surfaces. So, the solution Bi does not satisfy the boundary conditions $\left|E_{y}(x \rightarrow \pm \infty)<\infty\right|$, and, consequently, $a_{I I}^{2}=0$. So, we obtain the solution (9), where $a \equiv a_{I I}^{1}=0$. Its asymptotic in the intersection with the region III is

$E_{y}=\frac{a}{\sqrt{\pi}} \xi^{-1 / 4} \sin \left(\frac{2}{3} \xi^{2 / 3}+\frac{\pi}{4}\right)$.

The general solution in the region III is

$E_{y}=\frac{1}{\sqrt{k_{x}}}\left(a_{I I I}^{1} \exp i \int_{x_{1}}^{x} k_{x} d x^{\prime}+a_{I I I}^{2}\right.$ C.C. $)$.

To match with Eq. (9), we note that at $x \simeq x_{1} k_{x}^{2} \simeq U_{1}^{\prime} \cdot\left(x-x_{1}\right)$. Correlating it with Eq. (A1) we find

$a_{I I I}^{1,2}= \pm \frac{a}{\sqrt{\pi}} \frac{1}{2 i} e^{ \pm i \pi / 4}\left(2 K_{1}^{\prime}\right)^{1 / 6}$

and finally yield Eq. (10). In the intersection with region IV, where $k_{x}^{2}=-\left[\lambda_{0}\left(x-x_{0}\right)\right]^{-1}$, the asymptotic is

$$
\begin{aligned}
E_{y} & =\left[\lambda_{0}\left(x-x_{0}\right)\right]^{1 / 4}\left(a_{I I I}^{1} e^{i \varphi-2 i \sqrt{-\zeta}}\right. \\
& \left.+a_{I I I}^{2} e^{-i \varphi+2 i \sqrt{-\zeta}}\right)
\end{aligned}
$$

where $\zeta=\left(x-x_{0}\right) / \lambda_{0}$ and $\varphi=\int_{x_{1}}^{x_{0}} k_{x} d x$.

In region $\mathrm{IV}$, the equation takes the form

$E_{y}^{\prime \prime}-\frac{E_{y}}{\lambda_{0}\left(x-x_{0}\right)}=0$.

Its general solution at $x<x_{0}$ is

$E_{y}=\sqrt{-\zeta} \cdot\left[a_{I V}^{1-} \cdot \mathrm{J}_{1}(2 \sqrt{-\zeta})+a_{I V}^{2-} \cdot \mathrm{Y}_{1}(2 \sqrt{-\zeta})\right]$.

The asymptotic at $-\zeta \ll 1$ is

$E_{y}=-a_{I V}^{1-} \zeta-\frac{1}{\pi} a_{I V}^{2-}(1+2 \zeta \ln \sqrt{-\zeta})$,

and at $-\zeta \gg 1$ the asymptotic is

$$
\begin{aligned}
E_{y} & =\frac{1}{\sqrt{\pi}}(-\zeta)^{1 / 4}\left[a_{I V}^{1-} \cos \left(2 \sqrt{-\zeta}-\frac{3 \pi}{4}\right)\right. \\
& \left.+a_{I V}^{2-} \sin \left(2 \sqrt{-\zeta}-\frac{3 \pi}{4}\right)\right] .
\end{aligned}
$$

The general solution in the region IV at $x>x_{0}$ is

$E_{y}=\sqrt{-\zeta}\left[a_{I V}^{1+} \cdot \mathrm{I}_{1}(2 \sqrt{\zeta})+a_{I V}^{2+} \cdot \mathrm{K}_{1}(2 \sqrt{\zeta})\right]$.

The asymptotic at $\zeta \ll 1$ is

$E_{y}=a_{I V}^{1+} \zeta+\frac{1}{2} a_{I V}^{2+}(1+2 \zeta \ln \sqrt{\zeta})$ and at $\zeta \gg 1$ the asymptotic is

$E_{y}=\frac{1}{2}(\zeta)^{1 / 4}\left[\frac{1}{\sqrt{\pi}} a_{I V}^{1+} e^{2 \sqrt{\zeta}}+\sqrt{\pi} a_{I V}^{2+} e^{-2 \sqrt{\zeta}}\right]$.

The expressions (A3) and (A5) must be analytical continuations of each other. The relation between the coefficients with + and - upper indices can be established from the indenting the $x=x_{0}$ singularity. It should be bypassed in the upper half-plane of the $x$-variable, which corresponds to small absorption of the energy. It gives us relations

$a_{I V}^{1+}+\frac{i \pi}{2} a_{I V}^{2+}=-a_{I V}^{1-}, \quad a_{I V}^{2+}=-\frac{2}{\pi} a_{I V}^{2-}$.

Then, we compare Eq. (A4) with Eq. (A2) and find

$a_{I V}^{1-}=-a \sqrt{\lambda_{0}}\left(U_{1}^{\prime}\right)^{1 / 6} \cos \varphi$,

$a_{I V}^{2-}=-a \sqrt{\lambda_{0}}\left(U_{1}^{\prime}\right)^{1 / 6} \sin \varphi$,

and immediately yield Eqs. $(11,12)$.

In region $\mathrm{V}$ the solution of the Eq. (6) takes the form

$$
\begin{aligned}
E_{y} & =\frac{1}{\sqrt{\left|k_{x}\right|}}\left[a_{V}^{1} \exp \int_{x_{0}}^{x}\left|k_{x}\right| d x^{\prime}\right. \\
& \left.+a_{V}^{2} \exp \left(-\int_{x_{0}}^{x}\left|k_{x}\right| d x^{\prime}\right)\right] .
\end{aligned}
$$

At $x \simeq x_{0} \quad$ (in the intersection with region IV) $\left|k_{x}\right|^{2}=1 /\left[\lambda_{0}\left(x-x_{0}\right)\right]$. Correlating with Eq. (A6) after some algebra we obtain the expressions for the coefficients $a_{V}^{1,2}$ and finally the solution Eq. (13). On the other hand, near the point $x_{2}$, where $\left|k_{x}\right|^{2} \simeq U_{2}^{\prime} \cdot\left(x-x_{2}\right)$ the asymptotic is

$$
\begin{aligned}
E_{y} & =\left[2 K_{2}^{\prime}\left(x_{2}-x\right)\right]^{1 / 4} \cdot\left[a_{V}^{1} \exp \left(\psi+\frac{2}{3} \theta^{3 / 2}\right)\right. \\
& \left.+a_{V}^{2} \exp \left(-\psi-\frac{2}{3} \theta^{3 / 2}\right)\right],
\end{aligned}
$$

where $\theta=\left(x-x_{2}\right) / \lambda_{2}, \psi=\int_{x_{0}}^{x_{2}}\left|k_{x}\right| d x$.

Finally, we consider region VI, where Eq. (6) takes the form

$E_{y}^{\prime \prime}+U_{2}^{\prime} \cdot\left(x-x_{2}\right) E_{y}=0$.

Its general solution is

$E_{y}=a_{V I}^{1} \mathrm{Ai}(-\theta)+a_{V I}^{2} \mathrm{Bi}(-\theta)$.

Its asymptotic in the intersection with region $\mathrm{V}$ has the same form as Eq. (A7) with the coefficients

$a_{V I}^{1}=2 a\left(\frac{U_{1}^{\prime}}{U_{2}^{\prime}}\right)^{1 / 6} e^{-\psi} \sin \varphi$,

$a_{V I}^{2}=\frac{a}{2}\left(\frac{U_{1}^{\prime}}{U_{2}^{\prime}}\right)^{1 / 6} e^{\psi-i \varphi}$.

So, we have obtained the full solution in the WKBapproximation. 
Acknowledgements. The authors are grateful to V. A. Mazur and C. Othmer for the numerous discussions. The work of D. Yu. Klimushkin and P. N. Mager has been partially supported by Russian Foundation for Basic Research (RFBR) under grant 0405-64321. The work by K.-H. Glassmeier was financially supported by the German Ministerium für Bildung und Forschung and the Deutsches Zentrum für Luft- und Raumfahrt under contract 50OC0103. The work of P. N. Mager was supported by grant of Russian Science Support Foundation.

Topical Editor T. Pulkkinen thanks S. Fujita and M. Volwerk for their help in evaluating this paper.

\section{References}

Chen, L. and Cowley, S. C. : On fied line resonances of hydromagnetic Alfvén waves in a dipole magnetic field, Geophys. Res. Lett., 16, 895-899, 1989.

Cheng, A. F., Johnson, R. E., Krimigis, S. M., and Lanzerotti, L. J.: Magnetosphere, exosphere, and surface of Mercury, Icarus, 71, 430-440, 1987.

Dungey, J. W.: Electrodynamics of the outer atmosphere, Penn. State Univ. Ionos. Res. Lab. Sci. Rept., No. 69, 1954.

Fedorov, E. N., Mazur N. G., Pilipenko V. A., and Yumoto, K.: On the theory of field line resonances in plasma configurations, Phys. Plasmas, 2, 527-532, 1995.

Glassmeier, K.-H., Othmer, C., Cramm, R., Stellmacher, M., and Engebretson, M.: Magnetospheric field line resonances: A comparative planetology approach, Surv. Geophys., 20, 61-109, 1999.

Glassmeier, K.-H., Mager, P. N., and Klimushkin, D. Yu.: Concerning ULF pulsations in Mercury's magnetosphere, Geophys. Res. Lett., 30(18), 1928, doi:10.1029/2003GL017175, 2003.

Glassmeier, K.-H., Klimushkin, D. Yu., Othmer, C., and Mager, P. N.: ULF waves at Mercury: Earth, the giants, and their little brother compared, Adv. Space Res., 33, 1875-1883, 2004.

Hasegawa, A. and Chen, L.: Theory of magnetic pulsations, Space Sci. Rev., 16, 347-359, 1974.

Ip, W.-H.: The sodium exosphere and magnetosphere of Mercury, Geophys. Res. Lett., 13, 423-426, 1986.

Khurana, K. A.: ULF waves in other magnetospheres - Observations and possible source mechanisms, Ann. Geophys., 11, 973989, 1993.

Killen, R. M. and Ip, W.-H.: The surface-bounded atmospheres of Mercury and the Moon, Rev. Geophys., 37, 361-406, 1999.
Kim, E.-H. and Lee, D.-H.: Effects of heavy ions on ULF waves in the magnetosphere, EOS Trans. AGU, 85(47), Fall Meet. Supp., Abstract SM21A-0463, 2004.

Klimushkin, D. Yu.: Theory of azimuthally small-scale hydromagnetic waves in the axisymmetric magnetosphere with finite plasma pressure, Ann. Geophys., 16, 303-321, 1998.

Klimushkin, D. Yu. and Mager, P. N.: The structure of lowfrequency standing Alfvén waves in the box model of the magnetosphere with magnetic field shear, J. Plasma Phys., 70, 379-395, 2004.

Landau, L. D. and Lifshitz, E. M.: Electrodynamics of Continuous Media, Addison-Wesley Publishing Company, Inc., Reading, Massachusetts, 1960.

Leonovich, A. S. and Mazur, V. A.: Resonance excitation of standing Alfvén waves in an axisymmetric magnetosphere (Monochromatic oscillations), Planet. Space Sci., 37, 10951108, 1989.

Leonovich, A. S., and Mazur, V. A.: A theory of transverse smallscale standing Alfvén waves in an axially symmetric magnetosphere, Planet. Space Sci., 41, 697-717, 1993.

Leonovich, A. S., Mazur, V. A., and Senatorov, V. N.: Dispersion effects of MHD-waves in an inhomogeneous plasma, Issledovanija po geomagnetizmu, aeronomii i fizike Solntsa (in Russian), 66, 3-17, 1983.

Othmer, C., Glassmeier, K.-H., and Cramm, R.: Concerning field line resonances in Mercury's magnetosphere, J. Geophys. Res., 104, 10369-10378, 1999.

Russell, C. T.: ULF waves in the Mercury magnetosphere, Geophys. Res. Lett., 16, 1253-1256, 1989.

Southwood, D. J.: Some features of field line resonances in the magnetosphere, Planet. Space Sci., 22, 483-492, 1974.

Southwood, D. J. and Kivelson, M. G.: The effect of parallel inhomogeneity on magnetospheric hydromagnetic wave coupling, J. Geophys. Res., 91, 6871-6876, 1986.

Swanson, D. G.: Plasma Waves, Academic Press, Boston, 1989.

Tamao, T.: Transmission and coupling resonance of hydromagnetic disturbances in the non-uniform Earth's magnetosphere, Sci. Rept. Tohoku Univ., Series 5, Geophysics, 17, 43-72, 1965.

Timofeev, A. V.: Magnetoacoustic waves in a plasma in mirror systems, Fizika plazmy (in Russian), 18, 329-334, 1992.

Wedeken, U., Inhester, B., Korth, A., Glassmeier, K. H., Gendrin, R., Lanzerotti, L. J., Gough, H., Green., C. A., Amata, E., Pedersen, A., and Rostoker, G.: Groung satellite coordinated study of the April 5, 1979 events: flux association of energetic particles and associated magnetic pulsations, J. Geophys., 55, 120-133, 1984. 